\title{
Cystic adventitial disease of the popliteal artery (CADPA): a case report
}

\author{
Katarzyna Dudzinska', Wojciech Wlodarczyk', Eliza Pleban² \\ 'Department of General Surgery with Subunit of Oncological Surgery and Subunit of Vascular Surgery, Specialist Hospital, Pila, Poland \\ ${ }^{2}$ Department of Vascular Surgery, Institute of Haematology and Transfusion Medicine, Warsaw, Poland
}

\begin{abstract}
Cystic adventitial disease of the popliteal artery (CADPA) is a rare vascular, non-atherogenic disease. In most cases it occurs in the popliteal artery, between media and adventitia of the arterial wall. A cyst is a one- or multi-compartment structure filled with gelatinous content. By direct compression it leads to the destruction of the arterial wall and by narrowing or closing an artery it causes limb ischaemia resulting in intermittent claudication. This paper reports the case of a 29-year-old male with CADPA of the left popliteal artery, diagnostic process and treatment history.
\end{abstract}

Key words: cystic adventitial disease of the popliteal artery, CADPA, intermittent claudication

Acta Angiol 20I5; 21, 4: 136-140

\section{Case study}

29-year-old male patient contacted the GP complaining of the left calf pain persisting for three months, which gradually grew worse, especially during intense physical activity. The walking distance was 200 $-300 \mathrm{~m}$. The patient denied cardiac or metabolic co-morbidities which may have caused the discomfort. The patient did not connect the pain with any trauma in the area of the left popliteal fossa. He also denied smoking.

On physical examination the foot pulses at rest were palpable on both sides, slightly weakened on the left limb. After physical exercise, when the pain on the left calf occurred, the pulses on the posterior tibial and dorsal arteries disappeared. Colour Doppler ultrasound revealed a nodular structure involving the popliteal artery, causing reduced blood flow through the vessel (Fig. I). Because of a nonspecific image, the patient was referred for angio-CT with the initial diagnosis of popliteal artery aneurysm.

The CT scan revealed a cystic tumour surrounding the popliteal artery causing a significant narrowing of

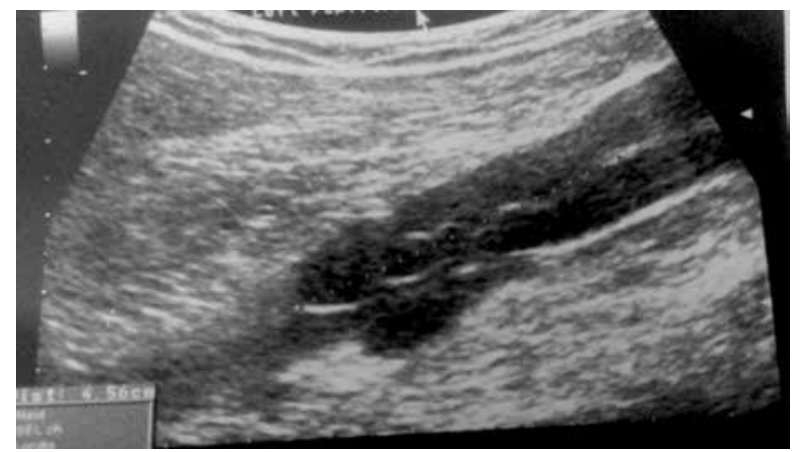

Figure I. An ultrasound image showing cystic adventitial disease of the popliteal artery

the artery lumen (Fig. 2). Due to increasing pain, the patient was qualified for open surgical treatment.

On abdomen supine position from the incision in the popliteal fossa a fibrous cyst, filled with mucous content, surrounding and compressing the popliteal artery was exposured.

A $3.5 \mathrm{~cm}$ long segment of the artery was resected together with the cyst and sent for histopathological 


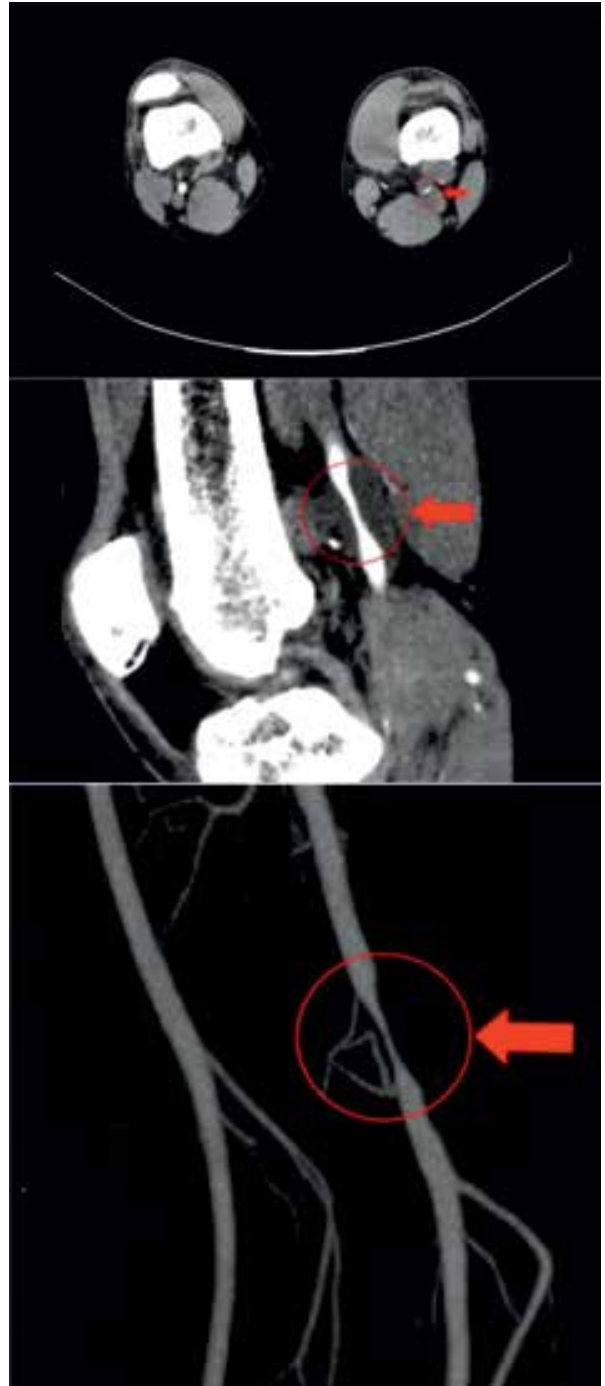

Figure 2. Angio CT reconstructions show narrowed lumen of the left popliteal artery and the outline of the cyst examination. The next step consisted in the reconstruction of the popliteal artery with the use of reversed saphenous vein. Histopathological examination confirmed the adventitial cyst of the artery in the specimen (Fig. 3).

The postoperative period was uneventful and the patient was discharged from hospital on the fifth day, with the recommendation of a control visit to the Vascular Surgery Outpatient Clinic.

The follow-up angio CT after six months revealed insignificant stenosis of the proximal anastomosis. Subsequent imaging showed no recurrence (Fig. 4). The patient is asymptomatic and remains under the control in the Vascular Surgery Outpatient Clinic.

Cystic adventitial disease (CAD) is a rare condition causing vascular non-atherosclerotic chronic limb ischaemia. It occurs in $0.1 \%$ of vascular diseases, mostly in males (F:M = I:I5), usually within the 4th and 5th decades of life [I, 2]. In most cases it affects the popliteal artery ( $85 \%$ of cases) and seldom external iliac, femoral, radial or ulnar arteries [3, 4]. CAD was first described in the external iliac artery in 1947 and next it was described in the popliteal artery in 1954 [5].

The aetiology still remains unknown. There are several hypotheses, i.e. local metabolic dysfunction in the artery wall or micro-traumas causing micro-bleeding and degenerative changes of the artery wall leading to accumulation of pathological material in the popliteal artery wall [6]. The most probable is thought to be the embryonic theory describing improper migration of mesenchymal cells during the process of organogenesis (between ca. week 15-22 of the foetal development), which take part in the creation of synovial capsules in the adjacent joints [7]. Considering the chemical composition of the cysts (mucopolysaccharides and mucoproteins) which is similar to the composition of

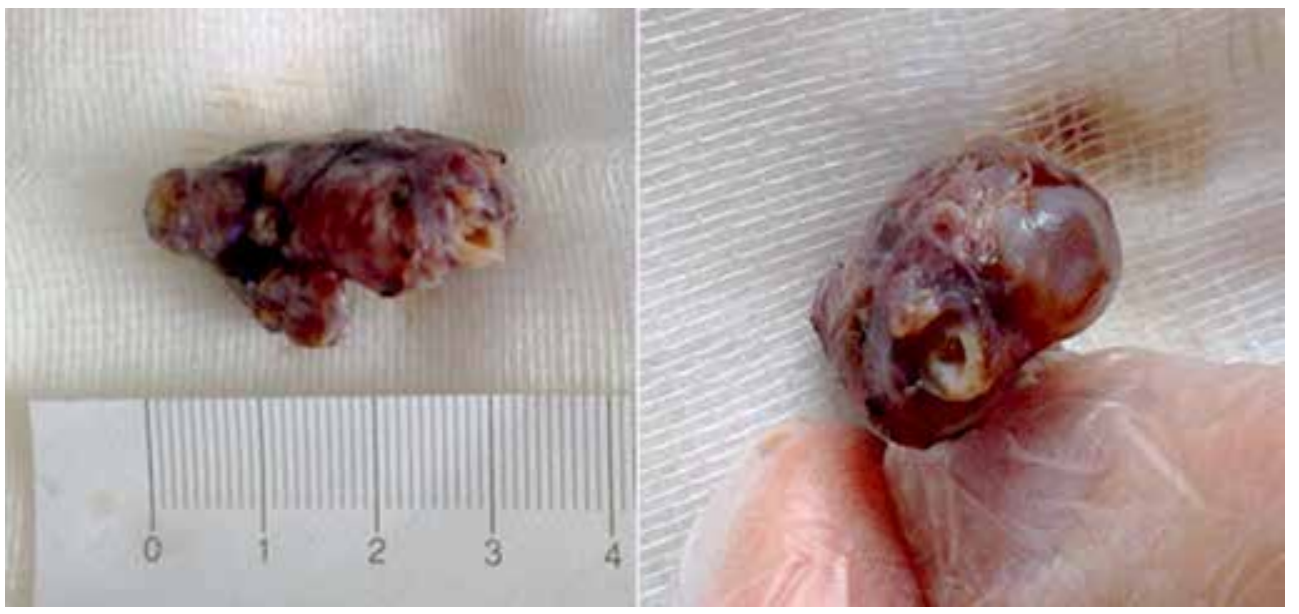

Figure 3. A fragment of the popliteal artery with the cyst 


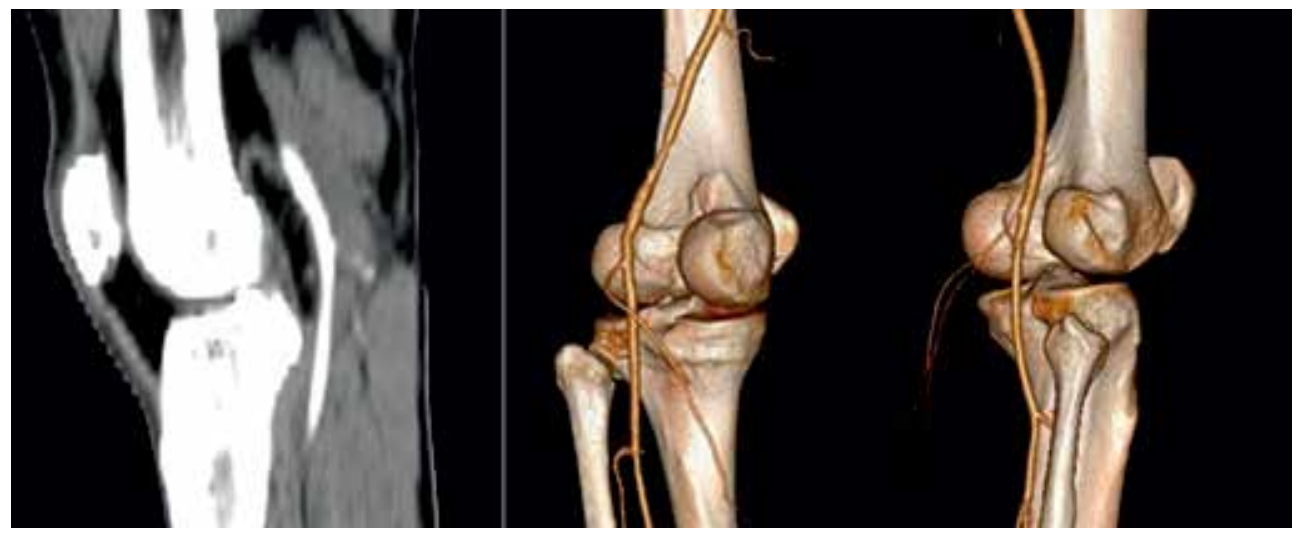

Figure 4. Angio CT images after surgical resection of the popliteal cyst and vessel reconstruction using the saphenous vein

joint synovial, and their localization close to large joints, this theory seems to be most convincing.

The most common symptom of CAD is quickly intensifying intermittent claudication of one lower limb in a young patient with no comorbidities such as atherosclerosis, hypertension, diabetes, hyperlipidaemia, systemic diseases and nicotinism. Limb pain subsides after a longer period of time and needs longer rest time than in case of intermittent claudication caused by atherosclerosis [8]. Rest pain occurs rarely, however, patients describe numbness and cramps in the leg. Physical examination reveals properly, symmetrically warm skin of the lower limb with no atrophy of skin appendages or ulcerations. The pulse on the suffering limb distally to the stenosis is weaker on palpation. $A B I$ is significantly lower in comparison with the contralateral limb and is reduced after physical effort [9]. What characterizes CADPA is Ishikawa sign, manifested by loss of pulses in foot arteries during knee flexion [10, I I].

The following diseases should be considered in differential diagnosis: atherosclerosis, Baker's cyst, posttraumatic lesion, popliteal artery aneurysm, Buerger's disease, popliteal artery entrapment syndrome (PAES), and even chronic venous insufficiency. The loss of pulse during plantar flexion of the foot when the knee is straightened is a useful symptom for the differentiation of CAD with the popliteal artery entrapment syndrome [8].

Primary diagnostic imaging in case of pain caused by a vascular disease is ultrasonography. Colour Doppler ultrasound reveals anechoic, hypoechoic mass in the artery wall, causing stenosis of the artery (Fig. I). The lesion leads to post-stenotic blood flow turbulences by increasing systolic blood pressure. It is common that the image of CAD is difficult to interpret (popliteal artery aneurysm, tumour of the popliteal fossa, Baker's cyst), and for this reason diagnostic imaging should be extended to other methods. Angiography shows smooth short segmental stenosis without any post-stenotic dilatation and no lesions in other vessels of the limb. CT and magnetic resonance angiography (MRA) show a single, few centimetres long cystic lesion that centrally compresses the vessel, giving the image of clepsydra-shaped stenosis (Fig. 2) or polycystic structure that ex-centrally bends and narrows the arterial lumen [9]. Due to precise imaging of vessels and the relations between the cyst and the surrounding structures, as well as the possibility of differential diagnostics of other popliteal artery diseases (atherosclerosis, aneurysm, popliteal entrapment syndrome - PAES), MRA is the examination of choice.

Invasive treatment options include open surgical and endovascular methods. At the moment, due to the best long-term results and lack of the recurrence, resection of the pathological vessel section and its reconstruction with the use of inversed saphenous vein or PTFE prosthesis is recommended, especially in case of tight stenosis or total occlusion. Resection of the cyst itself involves the risk of local recurrence and remains controversial. Ultrasound guided percutaneous cyst aspiration was described in individuals without significant stenosis or occlusion of vessels but due to the high percentage of disease recurrence (ca. $40 \%$ ) it is not widely recommended. The effectiveness rate of endovascular treatment (balloon angioplasty) is low (more than $67 \%$ recurrence rate); angioplasty of a structure which is not susceptible to stretching may lead to arterial wall rupture [9].

CADPA is a rare vascular disease. Appearance of intermittent claudication in young individuals with short history of symptoms, without other vascular diseases (diabetes, nicotinism, hypertension, dyslipidaemia) may raise suspicion of CAD. Unfortunately the very few existing articles which describe this condition, make diagnosis and treatment strategy choice difficult. For establishing the diagnosis, the following are helpful: taking a detailed history, physical examination and 
diagnostic imaging - ultrasound, angio CT and MRI. The best treatment option is segmental resection of the affected artery and its reconstruction with the use of a vein or vascular prosthesis.

\section{References}

I. Bunker S, Lauten GJ, Hutton JE ( 1981 ) Cystic adventitial disease of the popliteal artery. AJR Am J Roentgenol; 136: I209-1212.

2. Franca M, Pinto J, Machado R, Fernandez GC (2010) Bilateral adventitial cystic disease of the popliteal artery. Radiology; 255: 655-660.

3. Flanigan DP, Burnham SJ, Goodreau JJ, Bergan JJ (1979) Summary of cases of adventitial cystic disease of the popliteal artery. Ann Surg; 189: 165-175.

4. Peterson JJ, Kransdorf MJ, Bancroft LW, Murphey MD (2003) Imaging characteristics of cystic adventitial disease of the pe- ripheral arteries: presentation as soft tissue masses. AJR Am J Roentgenol; 180: 621-625.

5. Atkins $\mathrm{HJ}$, Key JA (1947) A case of myxomatous tumor arising in the adventitia of the left external iliac artery. $\mathrm{Br}$ J Surg; 34: 426.

6. Dwivedi AJ, Nguyen E, Cherukupalli C, Krishnasastry KV (2007) Cystic adventitial disease of the popliteal artery. MD All Specialties, May 25.

7. Levien LI , Benn CA (1998) Adventitial cystic disease: a unifying hypothesis. J Vasc Surg; 28: 193-205.

8. Igari K, Kudo T, Toyofuku T, Inoue Y (2015) Surgical Treatment of Cystic Adventitial Disease of the Popliteal Artery: Five Case Reports. Case Rep Vasc Med; 2015: 98468I.

9. Wright LB, Matchett WJ, Cruz CP et al (2004) Popliteal Artery Disease: Diagnosis and Treatment. Radiographics; 24: 467-479.

10. Ishikawa K (1987) Cystic adventitial disease of the popliteal artery and of other stem vessels in the extremities Jpn J Surg; 17: 22I-229.

II. Cassar K, Engeset J (2005) Cystic adventitial disease: a trap for the unwary. Eur J Vasc Endovasc Surg; 29: 93-96. 